\title{
Effects on Visual Recognition of Combined and Separate Ablations of the Entorhinal and Perirhinal Cortex in Rhesus Monkeys
}

\author{
M. Meunier, ${ }^{a}$ J. Bachevalier, ${ }^{b}$ M. Mishkin, and E. A. Murray \\ Laboratory of Neuropsychology, National Institute of Mental Health, Bethesda, Maryland 20892
}

\begin{abstract}
Performance on visual delayed nonmatching-to-sample was assessed in rhesus monkeys with combined and separate ablations of the perirhinal and entorhinal cortex, as well as in unoperated controls. Combined (i.e., rhinal cortex) lesions yielded a striking impairment on this task, one almost as severe as that seen after combined amygdalohippocampal removals that included some of this subjacent cortex (Mishkin, 1978; Murray and Mishkin, 1984). Ablations of the perirhinal cortex alone produced a deficit nearly as severe as that found after rhinal cortex lesions, whereas ablations of the entorhinal cortex alone produced only a mild deficit. Contrary to the conclusion from an earlier study (Murray and Mishkin, 1986), the present results demonstrate not only that damage limited to the rhinal cortex is sufficient to produce a severe loss in visual recognition, but also that such damage leads to a far greater loss than damage to any other single structure in the medial part of the temporal lobe.

IKey words: memory, hippocampus, amygdala, medial temporal lobe, delayed nonmatching-to-sample task, nonhuman primate]
\end{abstract}

A series of ablation studies in the early 1980 s (Zola-Morgan et al., 1982; Murray and Mishkin, 1984; Saunders et al., 1984) appeared to support an earlier conclusion (Mishkin, 1978) that a profound loss of recognition memory could be produced in monkeys by combined but not by separate removal of the amygdaloid complex and the hippocampal formation. Later, however, the possibility was raised that the accompanying damage to the underlying periallocortex, which includes, among others, both perirhinal and entorhinal cortex, might be largely responsible for the deficit that had been attributed to the combined amygdala and hippocampal lesions $(A+H)$, each of which damaged a different half of the rhinal cortical region (Horel et al., 1984, 1987; Murray et al., 1985, 1989; Murray and Mishkin, 1986; Zola-Morgan et al., 1989a,b, 1993). In an attempt to test that possibility, Murray and Mishkin (1986) examined the effects on visual recognition of removing the rhinal cortex (i.e., entorhinal and perirhinal cortex) in combination with either

\footnotetext{
Received Feb. 1, 1993; revised May 24, 1993; accepted June 1, 1993.

We thank Tinera Fobbs for histological processing of the tissue, Wendy Hadfield for help making the flat maps, and Maree Webster for generously providing advice and consultation during the preparation of the flat maps.

Correspondence should be addressed to Dr. E. A. Murray, Laboratory of Neuropsychology, NIMH, Building 49, Room IB80, Rethesda, MD 20892.

aPresent address: Laboratoire de Neurosciences Comportementales et Cognitives, Université de Bordeaux I, Talence, France.

'Present address: Department of Neurobiology and Anatomy, The University of Texas Medical School, Houston, TX.

Copyright (C) 1993 Society for Neuroscience $0270-6474 / 93 / 135418-15 \$ 05.00 / 0$
}

amygdalectomy or hippocampectomy. Monkeys with the amygdala plus rhinal cortex ablations $(A+R h)$ were found to be just as severely impaired as those with the complete removal, whereas monkcys with the hippocampus plus rhinal cortex ablations $(\mathrm{H}+\mathrm{Rh})$ were impaired far less, and, indeed, no more than those with hippocampectomy alone. It was therefore concluded that a rhinal cortex lesion was insufficient by itself to produce a severe memory impairment, and that its effect when combined with amygdalectomy was due to disconnection of the hippocampus from visual input, thereby resulting in a functional $\mathrm{A}+\mathrm{H}$ lesion. However, in light of recent architectonic and connectional studies (Amaral et al., 1987; Insausti et al., 1987) indicating that perirhinal cortex, in particular, extends more rostrally than had generally been appreciated before, the results of the Murray and Mishkin study can be interpreted in a very different way. According to this alternative interpretation, the removal in the $\mathrm{H}+\mathrm{Rh}$ group spared much more of the rostralmost portion of the perirhinal cortical field than did the removal in the $A+R h$ group. Thus, the more extensive rhinal cortex ablation in the $\Lambda+R h$ group, rather than its combination with amygdalectomy, might have accounted for the severe deficit this group sustained.

To reinvestigate this issue, we evaluated the effects on recognition memory of rhinal cortex ablations made in general conformity with the more recent anatomical descriptions provided by Amaral et al. (1987) and Insausti et al. (1987). In addition, to measure the contributions of the two different cytoarchitectonic components of this lesion, we compared the effects of removing the entorhinal cortex alone and the perirhinal cortex alone with those of the total ablation.

Preliminary reports of this work have appeared elsewhere (Murray et al., 1989; Meunier et al., 1990; Murray, 1992).

\section{Materials and Methods}

Subjects

The subjects were 19 naive rhesus monkeys (Macaca mulatta) of both sexes, weighing from 3.5 to $4.5 \mathrm{~kg}$ at the beginning of the study. They were housed individually in rooms with automatically regulated lighting (light/dark, $12 \mathrm{hr}: 12 \mathrm{hr}$ ). Monkeys were fed a diet of Primate Purina Chow supplemented with fruit; water was always available. During behavioral training, individual food rations were manipulated to ensure maximum feeding consistent with prompt responding in the testing apparatus.

\section{Apparatus and materials}

Training was conducted in a Wisconsin General Testing Apparatus inside a darkened, sound-shielded room. Additional sound masking was provided by a white-noise generator. During intertrial and retention intervals, an opaque screen separated the animal from the test tray. During the trials, a one-way vision screen separated the animal from the experimenter. The test tray contained a row of three food wells spaced $18 \mathrm{~cm}$ apart (center to center) and aligned $16 \mathrm{~cm}$ in front of the 
animal's cage. A single banana-flavored pellet ( $300 \mathrm{mg}$; P. J. Noyes Co.) or a half-peanut, concealed in one of the wells, served as the reward. Iest material consisted of 1120 different "junk" objects that varied widely in size, shape, texture, and color.

\section{Preoperative training}

Preliminary training. On the first day of adaptation, the animals were allowed to take food from the test tray. During subsequent days they were trained by successive approximation to displace a cardboard plaque covering the well in order to obtain the reward. Then a plaque or a nontest object was presented over the baited one of the three wells chosen in random order. This adaptation period, which was continued until the monkeys unhesitatingly displaced the baited plaque or nontest object, was normally completed in 4-6 d.

Delayed nonmatching-to-sample. Following adaptation, the monkeys were trained in delayed nonmatching-to-sample (DNMS) with trialunique objects (Mishkin and Delacour, 1975). On each trial, the central well was first covered by a sample object that the animal displaced in order to obtain the hidden food reward. Then, $10 \mathrm{sec}$ later, the sample object, now unbaited, and the baited novel object were presented for choice over the lateral wells. The left-right position of the novel object on the choice test followed a balanced pseudorandom order, and there was no correction for errors. Daily test sessions consisted of 20 trials separated by $30 \mathrm{sec}$ intertrial intervals. For each trial, a new pair of objects was drawn from the collection of 1120 test objects. The objects were used in order, so that only after all the objects had been used did they reappear, which occurred about once per month. Monkeys were trained to a criterion of 90 correct responses in 100 consecutive trials.

After learning the basic recognition task, the monkeys were assigned to four groups matched on the basis of their preoperative learning scores. One group remained unoperated (group $\mathrm{N} ; N=4$ ), whereas the three others received bilateral ablations of either the entorhinal cortex (group ERh; $N=4$ ), the perirhinal cortex (group PRh; $N=4$ ), or both (group $\mathrm{Rh} ; N=7$ ).

\section{Cytoarchitecture and intended lesions}

The rhinal cortex, located on the ventromedial aspect of the temporal lobe of the monkey brain, comprises both banks of the rhinal sulcus as well as some medially and laterally adjacent tissue (see Fig. 1). It is bordered rostrally by the temporal polar area TG, caudally by areas TF and TH of the parahippocampal gyrus, laterally by the visual area TE, which occupies the remainder of the inferior temporal cortex, and medially by the periamygdaloid and piriform cortices at anterior levels and the parasubiculum at posterior levels (von Bonin and Bailey, 1947).

In general, we followed the cytoarchitectonic descriptions of Amaral and his colleagues (Amaral et al., 1987; Insausti et al., 1987) in delimiting the perirhinal cortical field. The border between our ERh and PRh lesions was set at the fundus of the rhinal sulcus, although the actual border between the entorhinal and perirhinal cytoarchitectonic fields may be located either slightly medial or slightly lateral to the fundus at some levels. Our PRh lesions were thus intended to include both the lateral bank of the rhinal sulcus and about $2 \mathrm{~mm}$ of the cortex lateral to it (see Fig. 1). This field differs from the perirhinal cortex, areas 35 and 36, of Insausti et al. (1987) in two respects. First, although we used the same cytoarchitectonic criteria as they, we find that the lateral boundary of the perirhinal field in rhesus monkeys is located somewhat more medially than the lateral border of perirhinal cortex typically shown by these authors in cynomolgus monkeys. Second, for the temporal polar region, we retained the designation "area TG" of von Bonin and Bailey (1947) instead of "area 36p," because in the rhesus monkey the cytoarchitectonic features of this region differ from those of the perirhinal cortex. Thus, except for a small portion of TG located within $2 \mathrm{~mm}$ of the dorsal tip of the rhinal sulcus, this temporal polar area was excluded from our PRh lesions (see Fig. 1).

Our ERh lesion was intended to include both the medial bank of the rhinal sulcus and the cortex lying medial to it, that is, the two fields designated by Van Hoesen and Pandya (1975a) as the prorhinal and entorhinal cortices, respectively (see Fig. 1). The intended lesion also coincides with the entorhinal cortex as defined by Amaral and his colleagues (Amaral et al., 1987; Insausti et al., 1987), except that our lesion included a very small amount of tissue at the rostral end of the rhinal sulcus medial to the fundus that has been designated by these authors as belonging to perirhinal area 35 .

\section{Surgery}

Animals were anesthetized with ketamine hydrochloride $(10 \mathrm{mg} / \mathrm{kg}$ i.m.) followed either by sodium pentobarbital ( $25-35 \mathrm{mg} / \mathrm{kg}$, i.v.), which was supplemented throughout surgery as required, or by isoflurane (1$2 \%$, to effect). After induction of anesthesia, the animal was treated with atropine sulfate $(0.04 \mathrm{mg} / \mathrm{kg}$, i.m.) to reduce secretions. Surgery was carried out aseptically, and heart rate, respiration rate, and body temperature were monitored throughout the procedure.

In all cases, the zygomatic arch was removed, followed by removal of bone over the ventrolateral surface of the frontal and temporal lobes. The removal extended rostrally to the orbit, ventrally to the base of the temporal fossa, and caudally to the external auditory meatus. The dura mater was reflected to expose the brain, and the ablation (see below) was made with the aid of an operating microscope by aspiration of tissue with a small-gauge sucker. When the ablation was completed, the wound was closed in anatomical layers with silk or vicryl sutures. All animals received dexamethasone phosphate $(0.4 \mathrm{mg} / \mathrm{kg})$ and Di-Trim $(0.1 \mathrm{ml} /$ $\mathrm{kg}, 24 \%$ solution, i.m.) for $1 \mathrm{~d}$ before surgery and daily for 1 week after surgery to reduce swelling and prevent infection, respectively. Monkeys also received acetaminophen $(40 \mathrm{mg})$ for $3 \mathrm{~d}$ after surgery as an analgesic.

Rhinal cortex lesions (group $R h$ ). For the rostral part of this removal, the dura was cut in a crescent over the frontal and anterior temporal lobes, and the frontal lobe was elevated slightly, exposing the medial temporal pole. The pia mater on each lip of the rhinal sulcus was cauterized, after which both banks of the rostral portion of the sulcus were ablated as well as approximately $2 \mathrm{~mm}$ of tissue both medial and lateral to it. This portion of the lesion extended from the lateral sulcus along the rostral face of the temporal pole to the floor of the temporal fossa. When the removal was completed, the dura was sewn, and a second dural incision was made over the lateral temporal lobe. For this part of the surgery, the monkeys often received mannitol $(30 \% ; 30 \mathrm{cc}$, i.v., over $30 \mathrm{~min}$ ) to reduce brain volume and thereby increase the accessibility of the ventromedial rhinal cortex. With the monkey's head tilted at an angle of $120^{\circ}$ from the upright position, the base of the temporal lobe was elevated slightly until the ventral end of the first ablation was identified. The removal was then extended to the posterior tip of the rhinal sulcus, again including both banks of the sulcus as well as about $2 \mathrm{~mm}$ of cortex lateral to it. Medial to the sulcus, however, the removal in this caudal part of the ablation extended up to $5 \mathrm{~mm}$ farther than in the rostral part, in the attempt to include all of the entorhinal cortex at this level (see Fig. 1). The amygdala and hippocampus were left intact. Six monkeys received the rhinal cortex ablation bilaterally in one stage, and one monkey received it in two unilateral stages separated by a 2 week interval, the latter to provide a direct comparison with the ERh cases (see below).

Entorhinal and perirhinal cortex lesions (groups ERh and PRh). Ablations of either the entorhinal cortex alone or the perirhinal cortex alone were made through the same surgical approaches as described above. For the ERh lesions, the removal included the medial bank of the rhinal sulcus together with the cortex medial to it for a distance of $2 \mathrm{~mm}$ in the rostral part of the lesion, increasing, as described above, to $5 \mathrm{~mm}$ in the caudal part. For the PRh lesions, the removal included the lateral bank of the rhinal sulcus and the tissue extending approximately $2 \mathrm{~mm}$ lateral to it along its entire length. Because removal of entorhinal cortex while attempting to preserve perirhinal tissue required prolonged surgery, these lesions were always performed in two unilateral stages separated by a 2 week interval. Again to provide a direct comparison, one monkey given a PRh lesion was treated in the same way, but the remaining PRh cases were given bilateral lesions in one stage.

\section{Histology}

At the conclusion of behavioral testing, the operated animals were given a lethal dose of sodium pentobarbital and perfused intracardially with normal saline followed by $10 \%$ formalin. The brains were then removed, photographed, embedded in celloidin, and cut at $25 \mu \mathrm{m}$ in the coronal plane. Every twentieth section was stained with thionin, mounted, and coverslipped.

Lesion extent. For each experimental case, the volume of the lesion was estimated by plotting the lesion onto drawings of coronal sections taken at $1 \mathrm{~mm}$ intervals through a normal rhesus monkey brain. Entorhinal and perirhinal cortical fields were identified and their borders delineated on the standardized drawings. The extents of the entorhinal and perirhinal cortical areas in the normal brain were measured with a digitizer linked to a computer with software for determining the surface 


\begin{tabular}{|c|c|c|c|c|c|c|c|c|c|c|c|c|}
\hline \multirow[b]{2}{*}{ Case } & \multicolumn{4}{|c|}{ PRh damage } & \multicolumn{4}{|c|}{ ERh damage } & \multicolumn{4}{|c|}{ Total Rh damage } \\
\hline & $L \%$ & $R \%$ & $\bar{X} \%$ & $W \%$ & $L \%$ & $R \%$ & $\bar{X} \%$ & $W \%$ & $L \%$ & $R \%$ & $\bar{X} \%$ & $W \%$ \\
\hline \multicolumn{13}{|c|}{ Group Rh } \\
\hline $\mathrm{Rh}-1$ & 76 & 72 & 74 & - & 75 & 58 & 66 & - & 75 & 65 & 70 & 49 \\
\hline Rh-2 & 93 & 87 & 90 & - & 80 & 80 & 80 & - & 87 & 83 & 85 & 72 \\
\hline Rh-3 & 93 & 94 & 94 & - & 41 & 43 & 42 & - & 67 & 69 & 68 & 46 \\
\hline $\mathrm{Rh}-4$ & 75 & 79 & 77 & - & 75 & 71 & 73 & - & 75 & 75 & 75 & 57 \\
\hline $\mathrm{Rh}-5$ & 73 & 88 & 81 & - & 67 & 64 & 66 & - & 70 & 76 & 73 & 53 \\
\hline$R h-6$ & 95 & 100 & 97 & - & 84 & 68 & 76 & - & 89 & 84 & 87 & 75 \\
\hline Rh-7 & 75 & 83 & 79 & - & 94 & 70 & 82 & - & 85 & 77 & 81 & 65 \\
\hline$\overline{\boldsymbol{X}}$ & 83 & 86 & 85 & - & 74 & 65 & 69 & - & 78 & 76 & 77 & 60 \\
\hline \multicolumn{13}{|c|}{ Groups PRh and ERh } \\
\hline PRh-1 & 66 & 86 & 76 & 57 & 5 & 3 & 4 & 0 & - & - & 40 & 16 \\
\hline PRh-2 & 91 & 99 & 95 & 91 & 7 & 5 & 6 & 0 & - & - & 51 & 26 \\
\hline PRh-3 & 89 & 87 & 88 & 78 & 1 & 2 & 2 & 0 & - & - & 45 & 20 \\
\hline PRh-4 & 95 & 90 & 93 & 85 & 1 & 10 & 6 & 0 & - & - & 49 & 24 \\
\hline $\bar{X}$ & 85 & 91 & 88 & 78 & 3 & 5 & 4 & $\mathbf{0}$ & - & - & 46 & 21 \\
\hline ERh-1 & 33 & 18 & 25 & 6 & 82 & 67 & 75 & 55 & - & - & 50 & 24 \\
\hline ERh-2 & 51 & 70 & 61 & 36 & 50 & 80 & 65 & 40 & - & - & 63 & 38 \\
\hline ERh-3 & 33 & 28 & 31 & 9 & 65 & 77 & 71 & 50 & - & - & 51 & 26 \\
\hline ERh-4 & 94 & 18 & 56 & 17 & 98 & 80 & 89 & 79 & - & - & 73 & 47 \\
\hline$\overline{\boldsymbol{X}}$ & 53 & 33 & 43 & 17 & 74 & 76 & 75 & 56 & - & - & 59 & 34 \\
\hline
\end{tabular}

Estimated damage (in percentage of normal) to rhinal (Rh), entorhinal (ERh), and perirhinal (PRh) cortex in each subject of the three experimental groups. $L \%$, percentage damage in the left hemisphere; $R \%$, percentage damage in the right hemisphere; $\bar{X} \%$, average of $L \%$ and $R \% ; W \%=(L \% \times R \%) / 100$ (weighted index as defined by Hodos and Bobko, 1984).

area of the region outlined on each section, as well as the volume across sections (Jandel, Inc.). The volume of entorhinal and perirhinal cortical tissue removed in each experimental subject was similarly calculated and expressed as a percentage of normal (see Table 1). In a separate series of measurements in the normal animal, the areas of the entorhinal and perirhinal cortical fields, taken midway through the depth of the cortex, were calculated to be 78 and $74 \mathrm{~mm}^{2}$, respectively.

Flat maps. A two-dimensional reconstruction (flat map) of the cortex of the temporal lobe was made for two representative cases from each experimental group. For each hemisphere, tracings of layer IV were made from photographs $(9 \times)$ of 15-20 thionin-stained coronal sections taken at $1 \mathrm{~mm}$ intervals through the temporal lobe (Gattass and Gross, 1981; Fiorani et al., 1989; Webster et al., 1991). For each section through the lesion, the mediolateral extent of the removal was evaluated by comparison with two similarly processed brains of monkeys with lesions outside the temporal lobe, and the length of the missing segment of layer IV was then inserted in the tracing. Moderately stiff wire was bent around each layer IV tracing, and the cytoarchitectonic borders, estimated lesion extent, as well as the location of the lips and fundus of each sulcus were marked on the wire. The bent wires were then soldered at appropriate intervals to orthogonally oriented wires that represented the lips and fundi of sulci to create a three-dimensional model, which was then flattened. A two-dimensional map prepared in this manner from a control brain is shown in Figure $1 C$, and the representative cases from the three experimental groups are illustrated in Figures 2, 4, and 6 , respectively. Photomicrographs of Nissl-stained coronal sections from one case in each group are shown in Figures 3, 5, and 7, respectively.

Rhinal cortex lesions (group $R h$ ). The lesions sustained by all seven monkeys were bilaterally symmetrical, but in none of them was the lesion complete. The volume of damage averaged $77 \%$ (range, $68-87 \%$ ) of the total extent of rhinal cortex (Table 1). This included an average of $69 \%$ (range, $42-82 \%$ ) of the entorhinal cortex and $85 \%$ (range, 74 $97 \%$ ) of the perirhinal cortex. In all cases, the most medial part of the entorhinal cortex was spared, with the greatest sparing being located under the posterior half of the amygdala, where the entorhinal cortex reaches its maximal mediolateral extent. Also consistently spared was rostrolateral perirhinal cortex, the portion immediately behind the temporal polar area TG.

Encroachment of the lesions on areas TE and TF was generally slight.
Posterior ventral TE was damaged unilaterally in cases Rh-2 (see Fig. $2 B$ ), $\mathrm{Rh}-6$, and $\mathrm{Rh}-7$, and this damage extended to the anterior ventral portion of TE in Rh- 6 . The lesion encroached on area TF bilaterally in $\mathrm{Rh}-2$ (see Fig. $2 B$ ) and $\mathrm{Rh}-5$. The only other unintended damage was to the ventral part of the left amygdala in $\mathrm{Rh}-1$.

Perirhinal cortex lesions (group PRh). With one exception, the lesions were bilaterally symmetrical and substantial, averaging $88 \%$ (range, $76-$ $95 \%$ ) of the total volume of perirhinal cortex (Table 1). However, as in group $\mathrm{Rh}$, the rostrolateral part of perirhinal cortex was consistently left intact. The one asymmetrical case (PRh-1; see Fig. $4 A$ ) had greater sparing than the others on the left, in the region where the removals from the two surgical approaches were incompletely joined.

Inadvertent damage to areas $\mathrm{TE}$ and $\mathrm{TF}$ was again slight. Posterior ventral TE was damaged unilaterally in PRh-3 and $P R h-4$, and this damage extended to the anterior ventral portion of TE in PRh-4. The lesion encroached on area TF bilaterally in PRh-3 and PRh-4. Direct damage to the entorhinal cortex rarely exceeded $5 \%$ in either hemisphere (Table 1). Because transection of the white matter below the perirhinal cortex has been reported to result in disconnection of the entorhinal cortex from some of its neocortical inputs (Zola-Morgan et al., 1989b), this area was carefully examined in all four cases. In each case the damage was found to be limited to the superficial portions of the white matter.

Entorhinal cortex lesions (group ERh). The amount of damage averaged $75 \%$ (range, $65-89 \%$ ) of the total volume of entorhinal cortex (Table 1). The lesions in this group tended to be more asymmetrical than those in the others, differing between sides by an average of $19 \%$, as compared with average left-right differences of $5 \%$ and $9 \%$ for the $R h$ and PRh groups, respectively. As in the $R h$ group, the most medial part of the entorhinal cortex was consistently left intact. All animals had, in addition, some sparing of the medial bank of the rhinal sulcus, but this occurred bilaterally only in ERh-2.

Each ERh case sustained substantial inadvertent damage to the laterally adjacent perirhinal field, ranging from $25 \%$ to $61 \%$ of its total. This damage occurred consistently within the posterior half of the perirhinal cortex, extending to the anterior half only in one case (ERh-4) and only unilaterally (Table 1). Small infarctions were found in area TE in ERh-2 and ERh-3 (see Fig. 6B), whereas in ERh-1 and ERh-4 the lesion encroached slightly on area TF unilaterally (see Fig. 6A). Finally, 

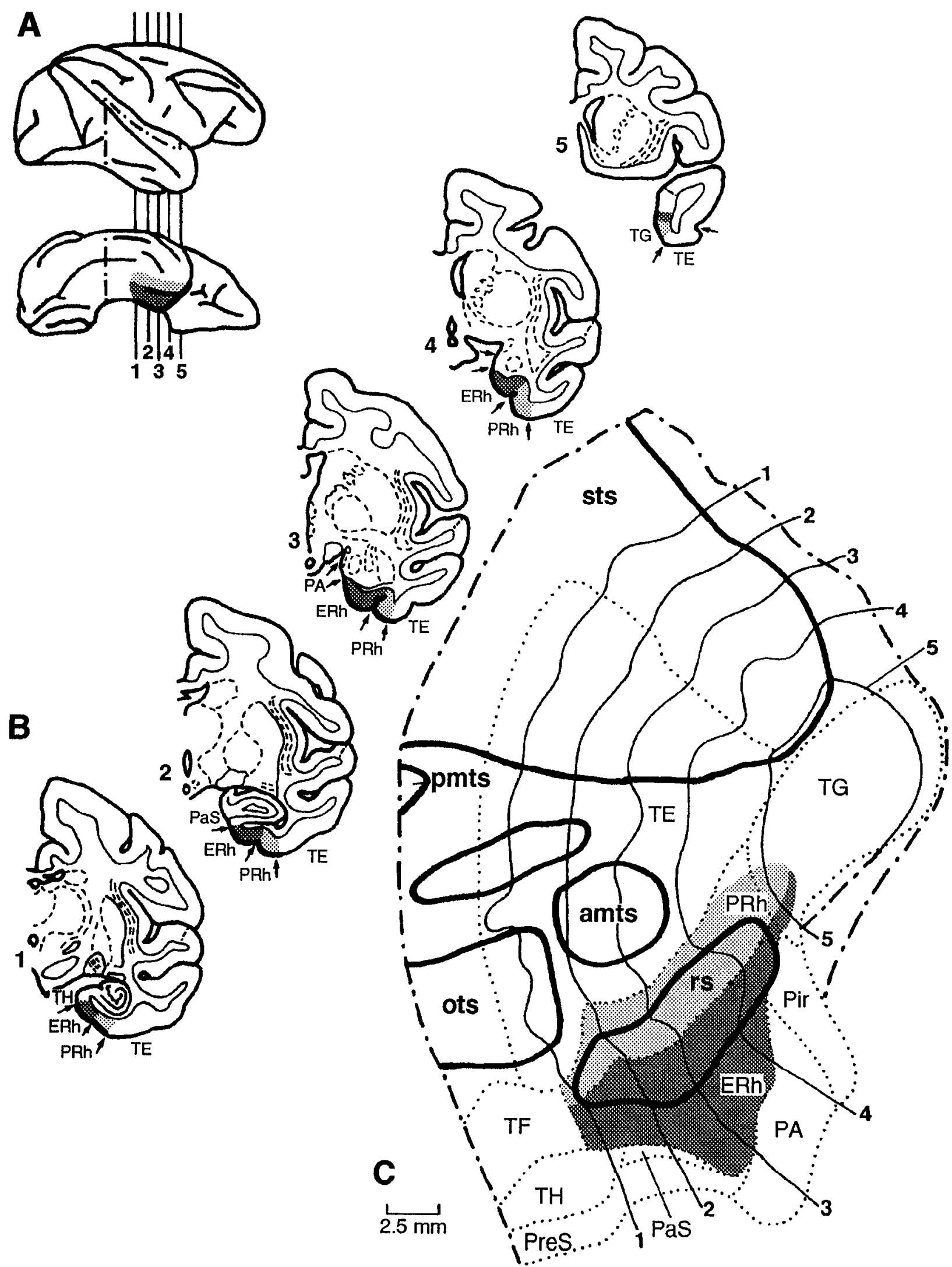

Figure 1. Location and extent of the intended lesions of the entorhinal cortex $(E R h)$ and perirhinal cortex $(P R h)$. Lesions of the rhinal cortex (Rh) are simply the combination of the other two. A, Lateral and ventral view of the right hemisphere of a rhesus monkey brain. Dot-dash line indicates the approximate borders (cut lines) of the flat map shown in $C$. Entorhinal cortex is shaded dark gray and perirhinal cortex is shaded light gray. $B$, Coronal sections through the brain showing the relationship of $A$ and $C$. Numerals $1-5$ match the levels shown in $A$ and $C$, and shading matches that shown in $A$ and $C$ as well. $C$, Flat map of the cortex in the temporal lobe (see text) showing location and extent of the entorhinal and perirhinal cortex. The map was constructed on the basis of measurements made on approximately 15 sections like those shown in $B$. Only five levels are illustrated for ease in viewing. Dot-dash lines indicate cut lines of map, thin solid lines indicate cortical layer 4 of sections $1-5$, thick lines indicate the lips of sulci, and dotted lines indicate the borders of cytoarchitectonic fields. The lateral and caudal boundaries of area IE are based on recent electrophysiological data from Boussaoud et al. (1991). TE, TG, TF, TH, cytoarchitectonic fields of von Bonin and Bailey (1947); PaS, parasubiculum; PreS, presubiculum; PA, periamygdaloid cortex; Pir, piriform cortex; $r s$, rhinal sulcus; ots, occipitotemporal sulcus; amts, anterior middle temporal sulcus; pmts, posterior middle temporal sulcus; sts, superior temporal sulcus. 

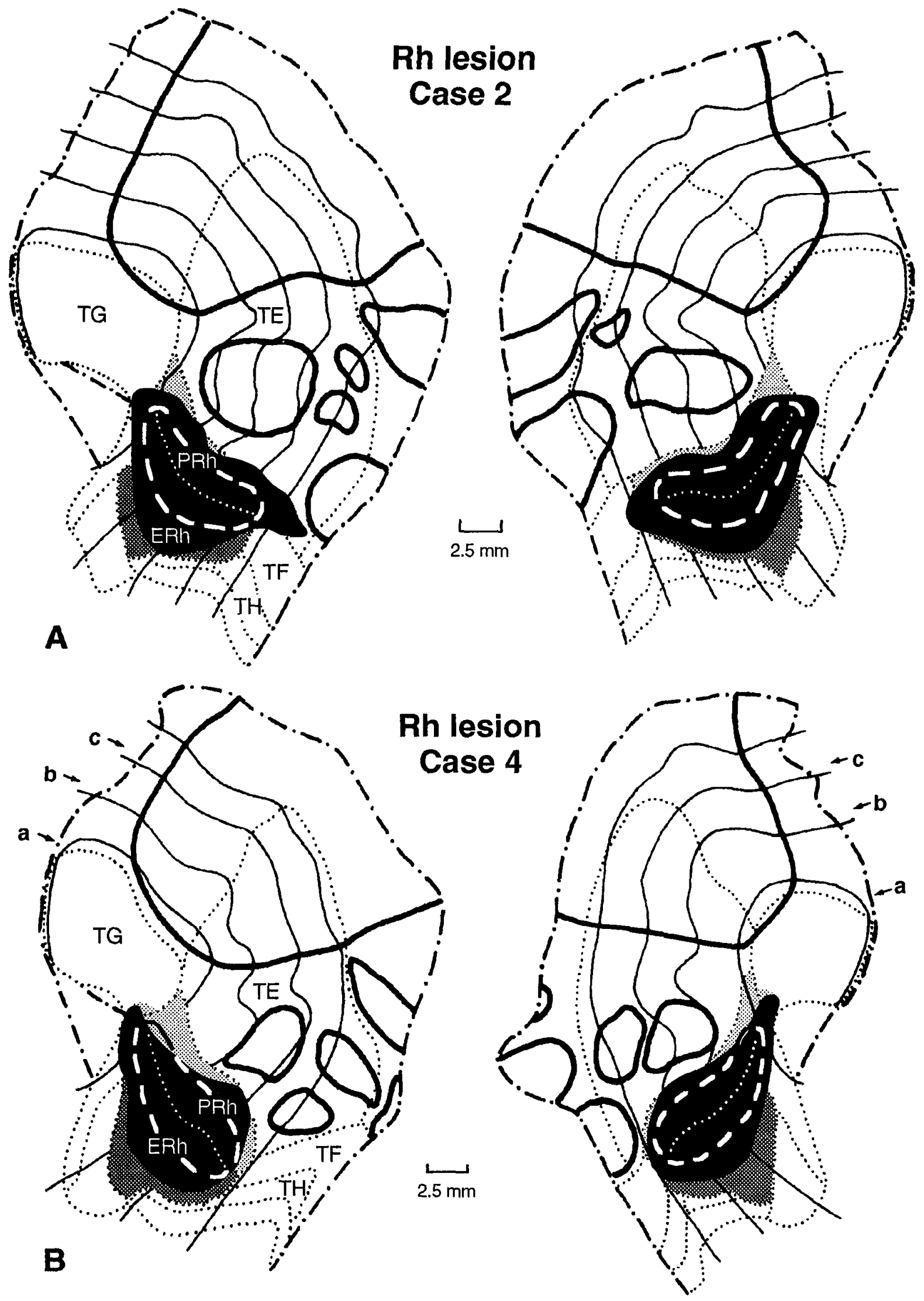

Figure 2. Location and extent of the rhinal cortex lesions in two cases, typical of the group, illustrated on flat maps of the temporal lobe. $A$, Removal (black area) and sparing (shaded areas) of rhinal cortex in Rh-2. B, Removal (black area) and sparing (shaded areas) in Rh-4. $a-c$ indicate the levels from which the photomicrographs in Figure 3 are taken. Conventions are as in Figure 1 . Compare and contrast with Figures 1 and 3. 

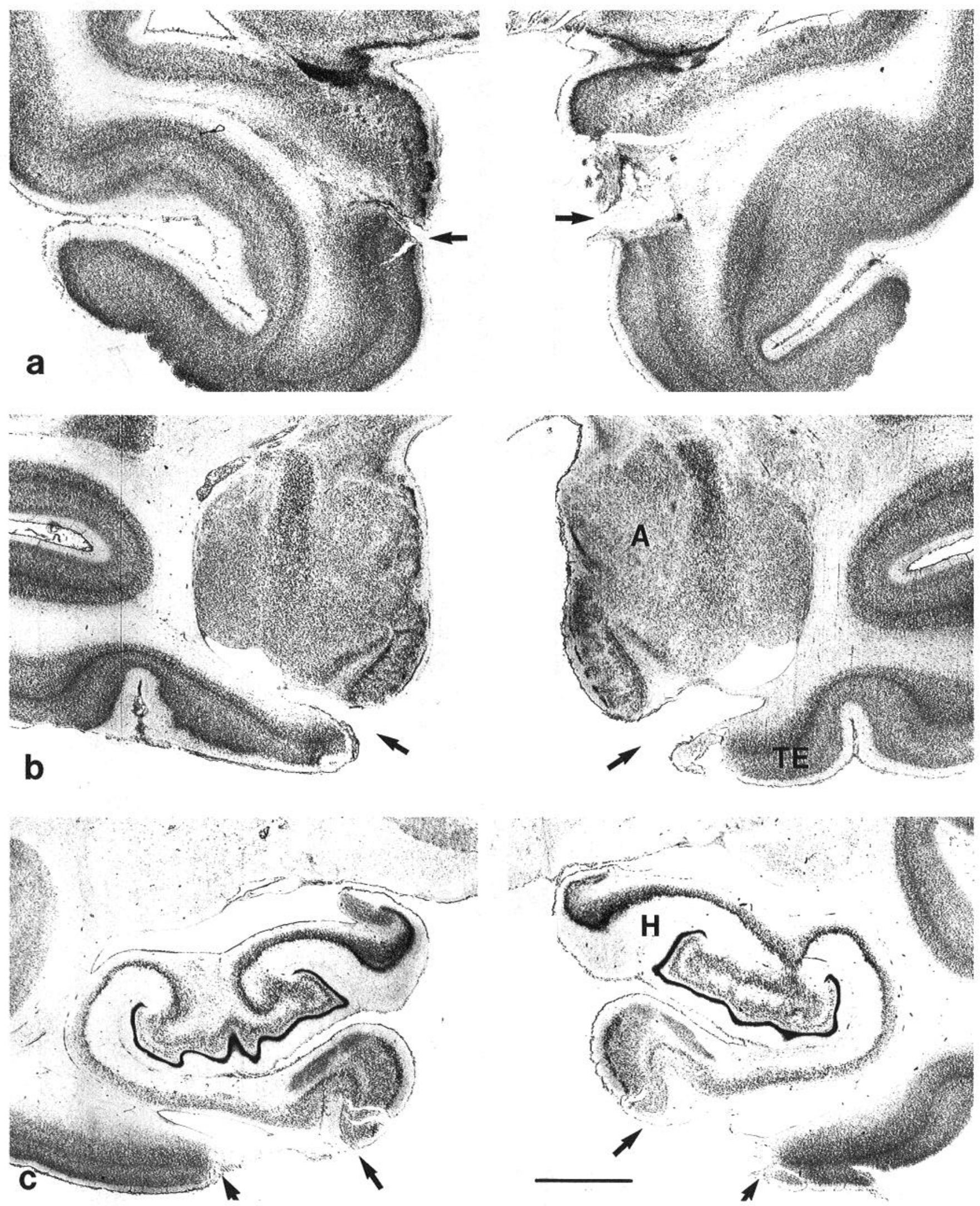

Figure 3. Photomicrographs of Nissl-stained coronal sections from a monkey with a rhinal cortex lesion (Rh-4). Arrows indicate site of lesion. Note sparing of the most medial portion of entorhinal cortex in level $b$. Sections $a-c$ correspond to the levels marked in Figure $2 B$. Compare and contrast with Figure 2. 

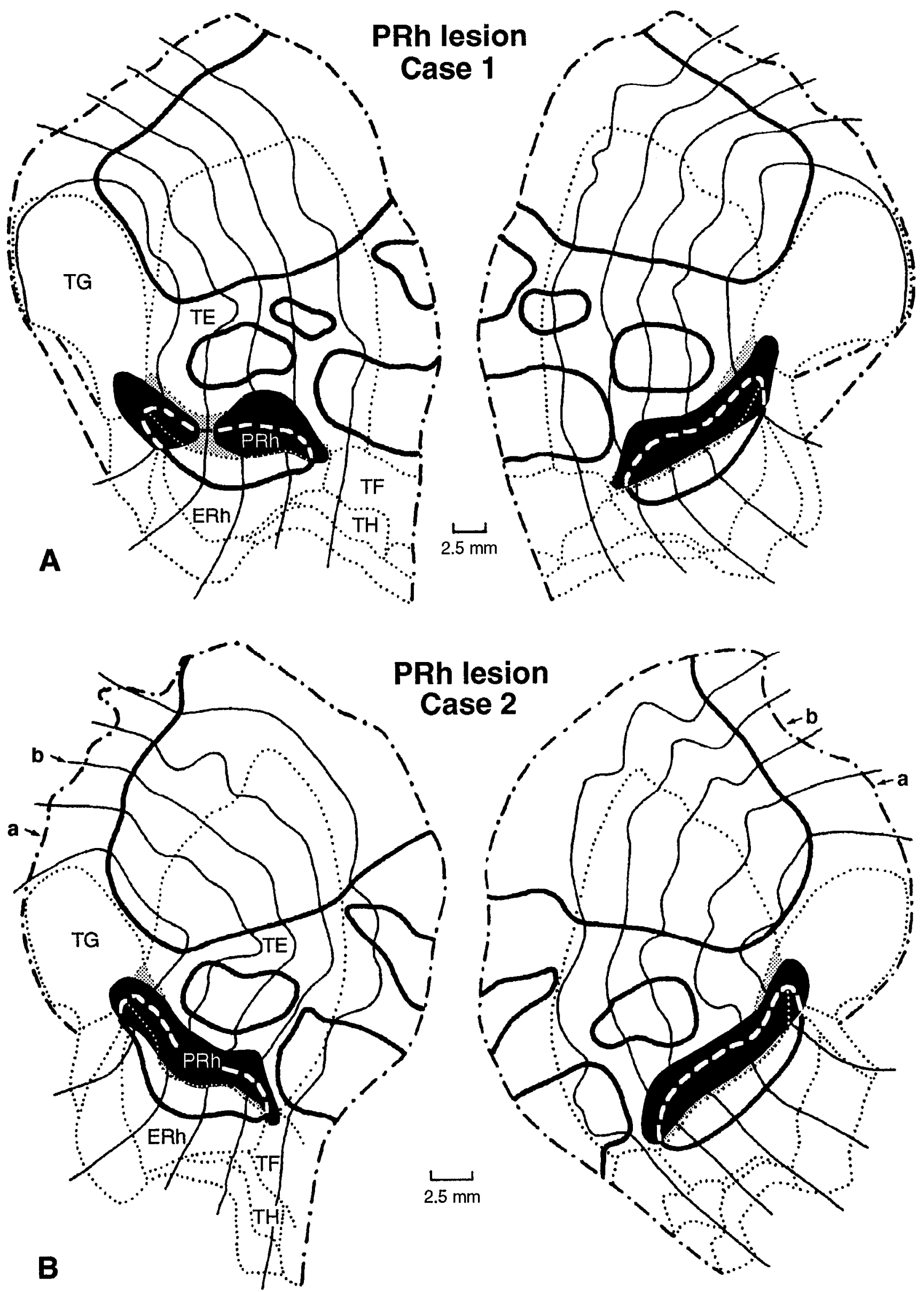

Figure 4. Location and extent of the perirhinal cortex lesions in two cases, typical of the group, illustrated on flat maps of the temporal lobe. Conventions are as in Figures 1 and 2. Levels $a$ and $b$ in $B$ correspond to the sections shown in Figure 5 . 

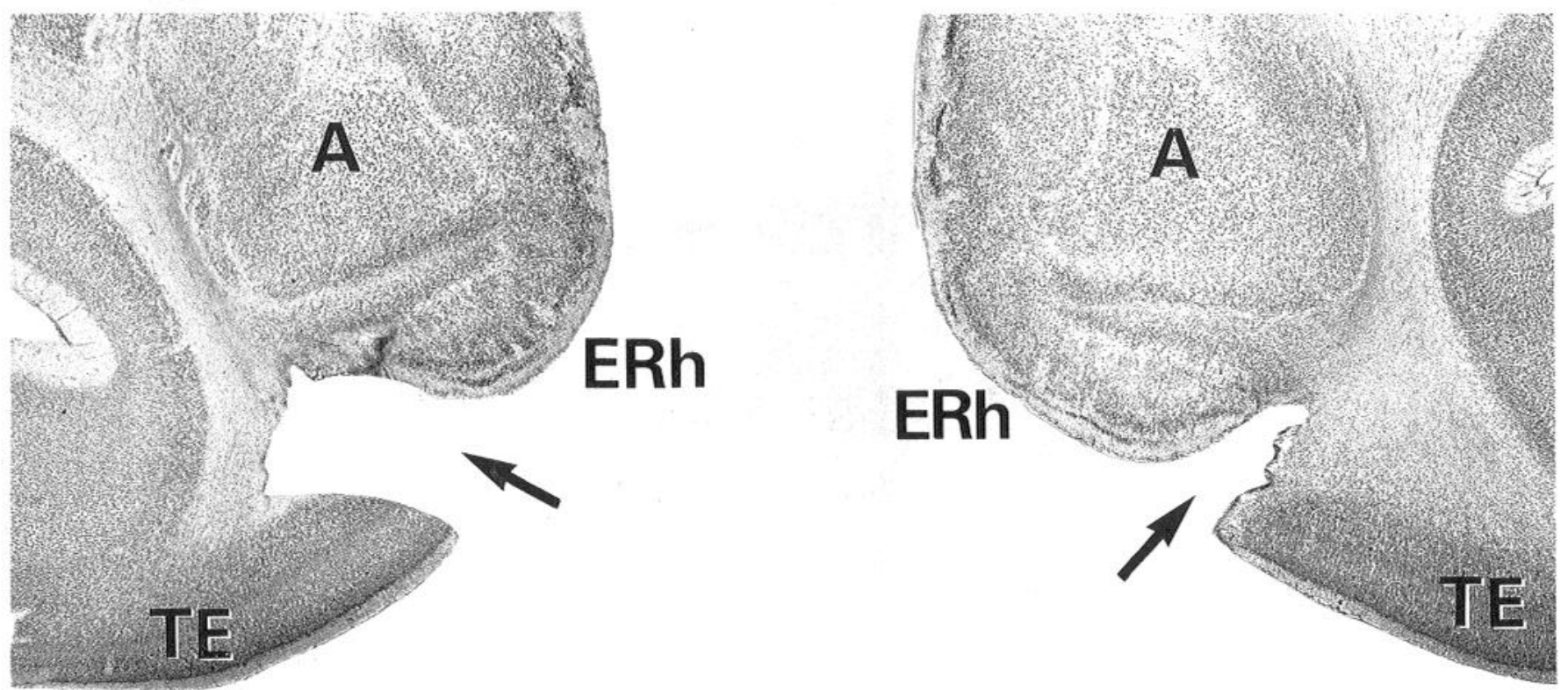

\section{a}
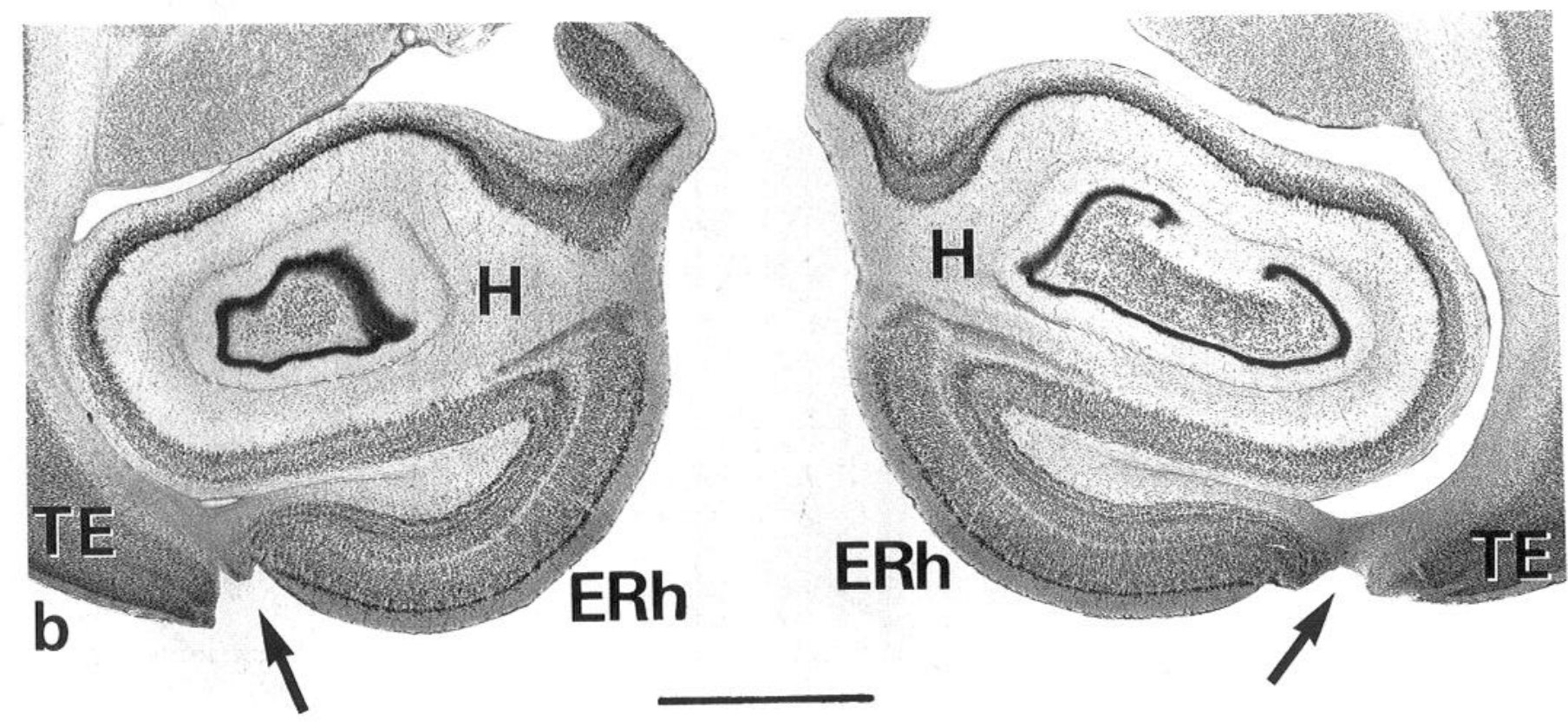

Figure 5. Photomicrographs of Nissl-stained coronal sections from a monkey with a perirhinal cortex lesion (PRh-2). Conventions are as in Figure 3. Sections $a$ and $b$ correspond to the levels shown in Figure $4 B$. Compare and contrast with Figure 4.

inadvertent damage to the ventral part of the amygdala occurred in ERh-2 and ERh-4 unilaterally.

\section{Postoperative testing}

Approximately 2 weeks after surgery, or after a 2 week rest period for the controls, the monkeys were retrained to criterion on the basic DNMS task. Following relearning they were given a performance test adapted from Gaffan (1974) in which, first, the delay between sample presentation and choice test was lengthened in stages from the initial delay of $10 \mathrm{sec}$ to 30,60 , and, finally, $120 \mathrm{sec}$, and then the list of sample objects to be remembered was increased in steps from the original single object to 3,5 , and, finally, 10 objects. In the list-length tests, the sample objects were presented one at a time at $20 \mathrm{sec}$ intervals, and then each sample was paired successively with a different novel object, also at $20 \mathrm{sec}$ intervals. Consequently, the minimal retention interval for each choice trial was $20 \mathrm{sec}$ multiplied by the length of the list. For each delay interval, the monkeys received five consecutive daily sessions of 20 trials each, and, for each list length, they received five consecutive daily sessions of 30 trials each.

\section{Results}

Preoperatively, the 19 monkeys attained criterion on DNMS in an average of 120 trials (range, 0-260) and 33 errors (range, 0 78). A parametric one-way ANOVA confirmed that the four groups that were formed (N, Rh, PRh, and ERh) did not differ from each other in these initial learning scores.

Postoperative relearning scores (Table 2, Fig. 8) differed significantly among groups [Kruskal-Wallis one-way ANOVA, $H(3)$ 

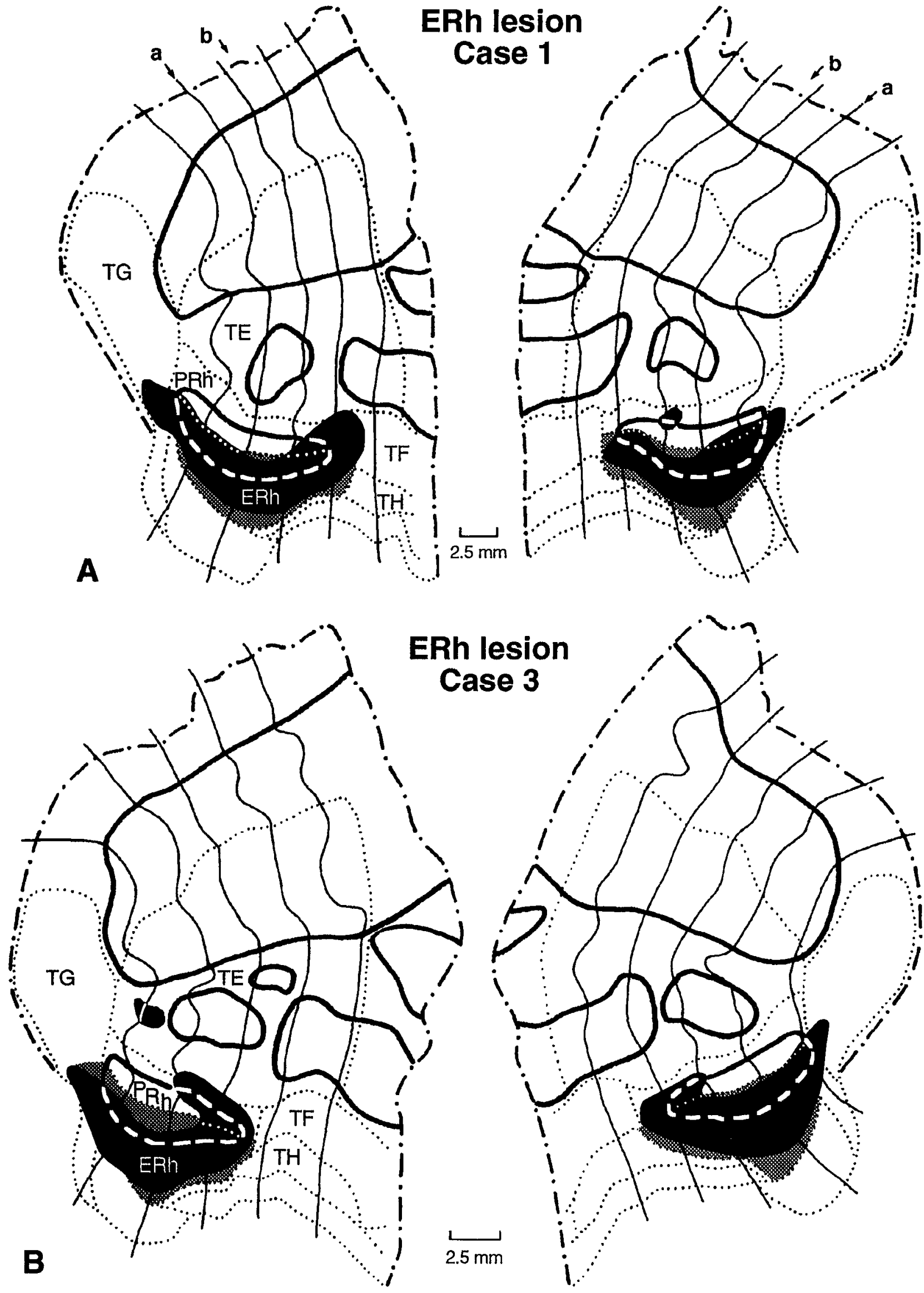

Figure 6. Location and extent of the entorhinal cortex lesions in two cases, typical of the group, illustrated on flat maps of the temporal lobe. Conventions are as in Figures 1 and 2. Levels $a$ and $b$ in $A$ correspond to the sections shown in Figure 7. 

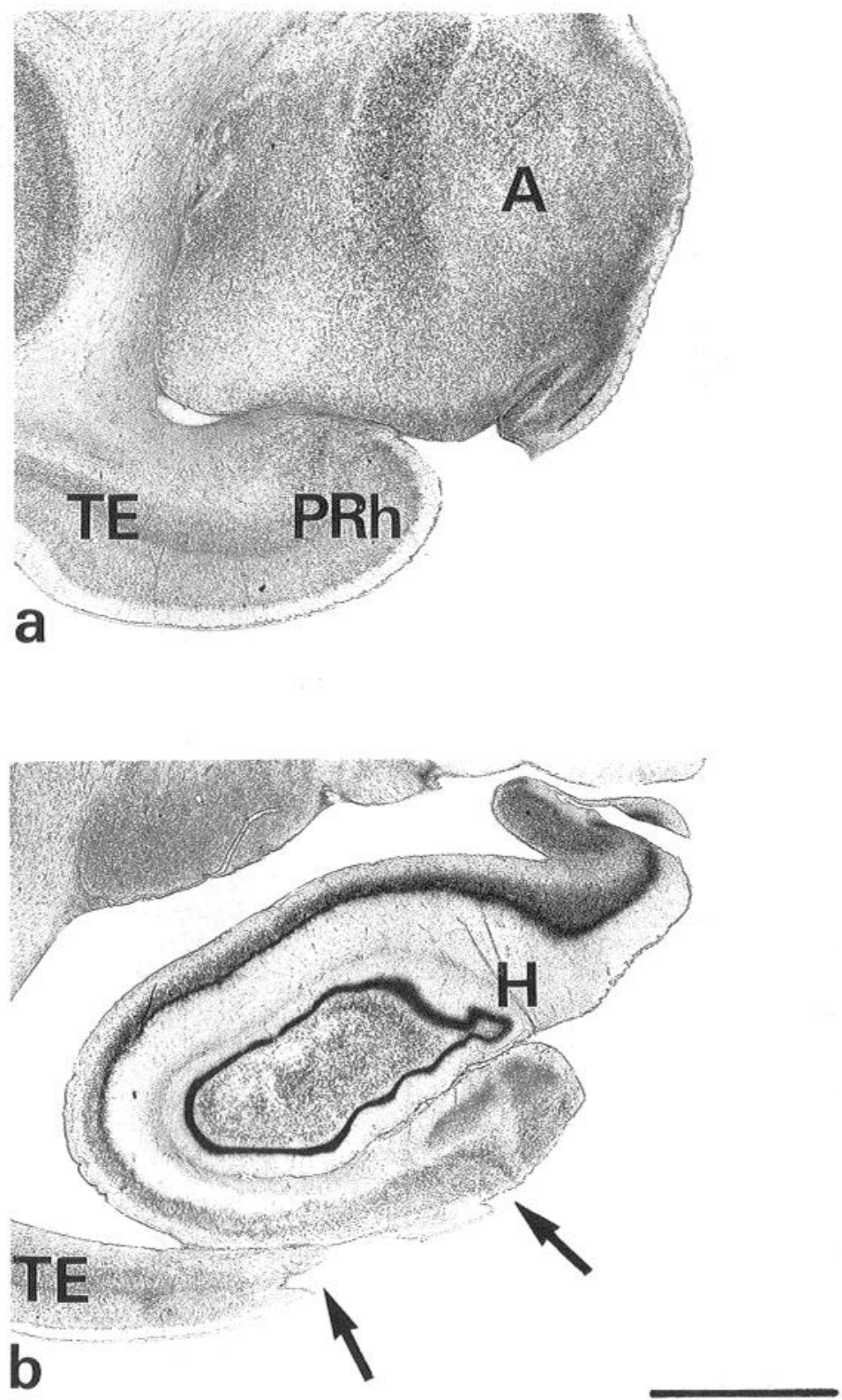
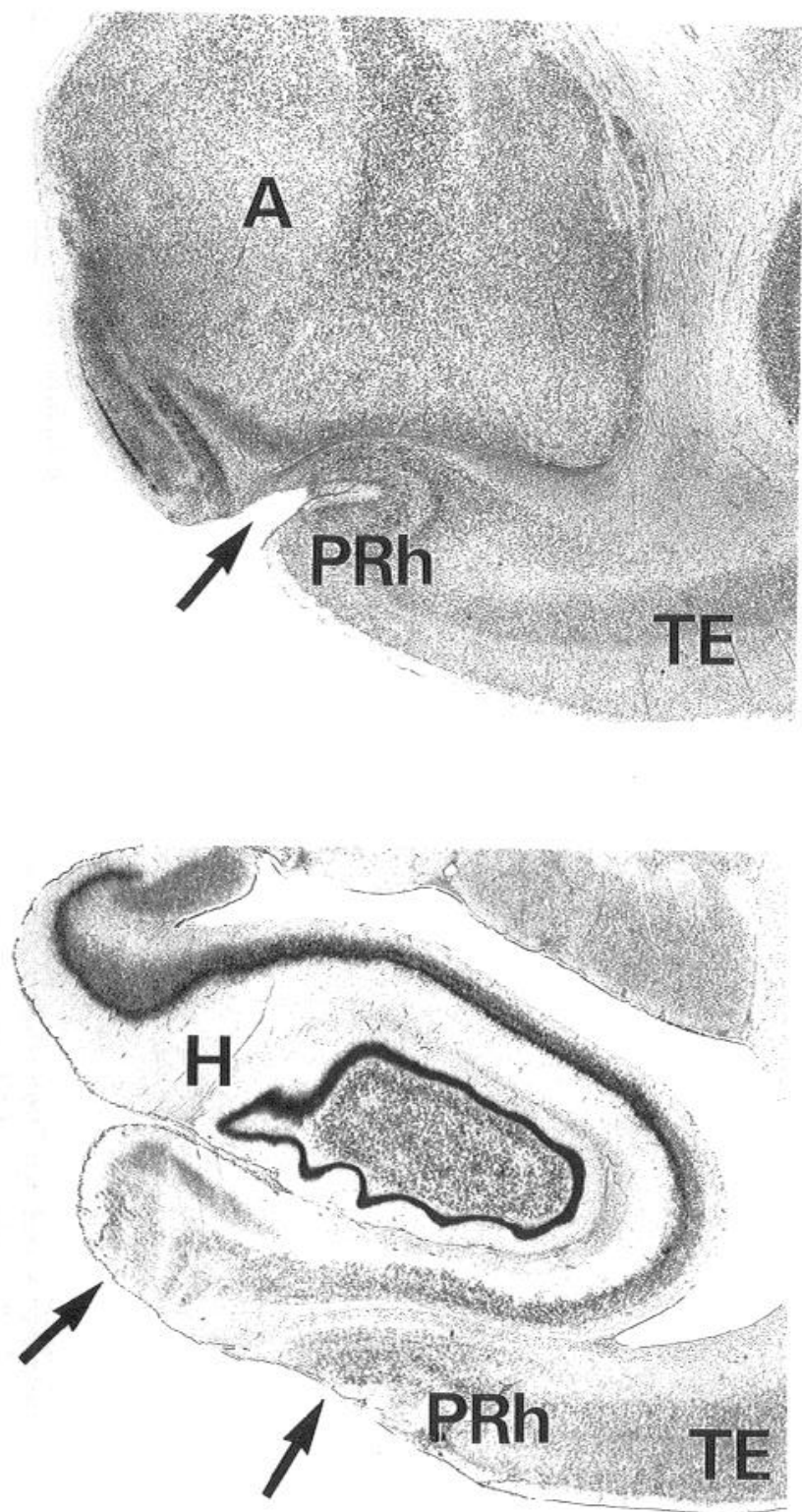

Figure 7. Photomicrographs of Nissl-stained coronal sections from a monkey with an entorhinal cortex lesion (ERh-1). Conventions are as in Figure 3. Sections $a$ and $b$ correspond to the levels shown in Figure $6 \mathrm{~A}$. Compare and contrast with Figure 6.

$=13.40, p=0.004]$. Whereas the controls relearned the task immediately, the $\mathrm{Rh}$ group required a mean of 497 trials and 140 errors, or about four times the number of trials they had taken preoperatively (Mann-Whitney $U$ test; Rh vs N: $U=0$, $p=0.007)$. The PRh group required an average of 380 trials and 82 errors to relearn, thus exhibiting a deficit roughly comparable to that of the Rh group (PRh vs $\mathrm{N}: U=0, p=0.014$; PRh vs Rh: $p=\mathrm{NS}$ ). By contrast, the ERh group was not significantly impaired (ERh vs N: $p=\mathrm{NS}$; ERh vs Rh: $U=0$, $p=0.003$; ERh vs PRh: $U=2, p=0.057$ ).

The groups also differed markedly on the postoperative performance test (Table 2, Fig. 9). Monkeys in the Rh group showed extremely rapid forgetting, obtaining an average of only $67 \%$ correct responses over the six conditions as compared with $93 \%$ for the controls. Monkeys in the PRh group averaged $78 \%$ correct responses, and those in the ERh group averaged $85 \%$ correct responses. The mean scores on the performance test were analyzed by a $4 \times 7$ ANOVA with one repeated measure (scores at the $10 \mathrm{sec}$ delay were included). Both main effects were significant [lesions: $F=46.30, \mathrm{df}=3,15, p<0.001$; conditions: $F$ with conservative $\mathrm{df}(1,15)=24.47, p<0.001$ ], as was their interaction $[F$ with conservative $\mathrm{df}(3,15)=4.21, p<0.05]$. Pairwise comparisons of the scores only on the delay conditions showed that all three experimental groups differed significantly from the normal controls (Tukey's HSD test; Rh vs N: $p<$ 0.001 ; PRh vs N: $p=0.009$; ERh vs $\mathrm{N}: p=0.026$ ). In addition, group $\mathrm{Rh}$ performed significantly more poorly than each of the other lesion groups (Rh vs PRh: $p=0.012$; Rh vs ERh: $p=$ 0.005 ), but the latter two did not differ from each other. A slightly different pattern of results emerged from pairwise comparisons on the list length conditions. Here, both groups Rh and PRh differed not only from the normal controls (Rh vs N: $p<$ 


\begin{tabular}{|c|c|c|c|c|c|c|c|c|c|c|c|c|}
\hline \multirow[b]{2}{*}{ Case } & \multicolumn{2}{|l|}{ Preop } & \multicolumn{2}{|c|}{ Postop } & \multicolumn{4}{|c|}{ Delays (sec) } & \multicolumn{3}{|c|}{ List lengths } & \multirow{2}{*}{$\begin{array}{l}\text { Aver- } \\
\text { age }\end{array}$} \\
\hline & $T$ & $(E)$ & $T$ & $(E)$ & 10 & 30 & 60 & 120 & 3 & 5 & 10 & \\
\hline N-1 & 0 & 0 & 0 & 0 & 92 & 96 & 95 & 96 & 93 & 95 & 84 & 93 \\
\hline $\mathrm{N}-2$ & 260 & 56 & 0 & 0 & 95 & 96 & 94 & 96 & 93 & 93 & 83 & 93 \\
\hline $\mathrm{N}-3$ & 120 & 35 & 0 & 0 & 98 & 96 & 97 & 94 & 89 & 89 & 79 & 91 \\
\hline $\mathrm{N}-4$ & 40 & 14 & 0 & 0 & 93 & 99 & 96 & 91 & 97 & 95 & 85 & 94 \\
\hline $\overrightarrow{\boldsymbol{X}}$ & 105 & 26 & 0 & 0 & 95 & 97 & 96 & 94 & 93 & 93 & 83 & 93 \\
\hline $\mathrm{Rh}-1$ & 220 & 78 & 340 & 115 & 90 & 75 & 66 & 59 & 69 & 57 & 57 & 64 \\
\hline Rh-2 & 220 & 53 & 560 & 123 & 91 & 82 & 68 & 63 & 72 & 67 & 63 & 69 \\
\hline $\mathrm{Rh}-3$ & 20 & 3 & 340 & 102 & 91 & 75 & 81 & 71 & 79 & 65 & 62 & 72 \\
\hline $\mathrm{Rh}-4$ & 20 & 6 & 540 & 150 & 90 & 83 & 76 & 67 & 75 & 68 & 59 & 71 \\
\hline Rh-5 & 200 & 51 & 720 & 228 & 90 & 67 & 68 & 70 & 67 & 56 & 53 & 64 \\
\hline $\mathrm{Rh}-6$ & 80 & 20 & 740 & 221 & 90 & 68 & 60 & 77 & 64 & 61 & 59 & 65 \\
\hline Rh-7 & 140 & 50 & 240 & 38 & 91 & 82 & 76 & 62 & 59 & 57 & 65 & 67 \\
\hline$\overline{\boldsymbol{X}}$ & 129 & 37 & 497 & 140 & 90 & 76 & 71 & 67 & 69 & 61 & 60 & 67 \\
\hline PRh-1 & 60 & 21 & 600 & 123 & 91 & 84 & 80 & 72 & 76 & 81 & 68 & 77 \\
\hline PRh-2 & 180 & 41 & 600 & 133 & 90 & 83 & 80 & 77 & 78 & 81 & 78 & 79 \\
\hline PRh-3 & 140 & 40 & 220 & 59 & 91 & 92 & 88 & 81 & 69 & 67 & 72 & 78 \\
\hline PRh-4 & 40 & 13 & 100 & 14 & 93 & 95 & 88 & 87 & 79 & 68 & 53 & 78 \\
\hline $\boldsymbol{X}$ & 105 & 29 & 380 & 82 & 91 & 89 & 84 & 79 & 76 & 74 & 68 & 78 \\
\hline ERh-1 & 160 & 37 & 200 & 34 & 90 & 96 & 88 & 90 & 83 & 85 & 85 & 88 \\
\hline ERh-2 & 100 & 21 & 140 & 38 & 91 & 76 & 75 & 79 & 83 & 88 & 82 & 81 \\
\hline ERh-3 & 1200 & 26 & 0 & 0 & 91 & 93 & 96 & 91 & 95 & 95 & 90 & 93 \\
\hline ERh-4 & 160 & 56 & 20 & 3 & 90 & 85 & 76 & 81 & 85 & 81 & 72 & 80 \\
\hline $\bar{X}$ & 135 & 35 & 90 & 19 & 91 & 88 & 84 & 85 & 87 & 87 & 82 & 85 \\
\hline
\end{tabular}

Scores are trials $(T)$ and errors $(E)$, preceding criterion both pre- and postoperatively on the basic recognition task, and percentage correct responses on extended delays and list lengths presented postoperatively. Average is the mean percentage correct responses (six conditions; that is, excluding the $10 \mathrm{sec}$ delay); $\mathrm{N}$, normal controls; Rh, bilateral lesion of the rhinal cortex (i.e., both the ento- and perirhinal cortices); PRh, bilateral lesion of the perirhinal cortex alone; ERh, bilateral lesion of the entorhinal cortex alone.

$0.001 ;$ PRh vs N: $p<0.001$ ), but also from group ERh (Rh vs ERh: $p<0.001$; PRh vs ERh: $p=0.007$ ). Indeed, on the listlength tests, group ERh no longer exhibited a reliable deficit, although the difference in the scores of groups PRh and $\mathrm{Rh}$ fell just short of significance (Rh vs PRh: $p=0.062$ ).

No within-group correlations between extent of damage and behavioral measures (errors to relearn DNMS or mean percentage correct responses on the performance test) attained significance. In group $\mathrm{Rh}$, for example, the extent of rhinal cortical damage $(W \%)$ was only weakly correlated with the errors accrued in relearning (Spearman's $r=0.286, p=N S$ ) and with the mean score on the performance test $(r=-0.107, p=$ NS). [If all 15 animals in the experimental group are considered together, however, there is a significant negative correlation between the magnitude of rhinal cortical damage $(W \%)$ and performance test scores $(r=-0.636, p<0.01)$.]

\section{Discussion}

Bilateral ablations of the rhinal cortex (i.e., the entorhinal and perirhinal cortical fields combined) produced a striking deficit in visual recognition memory as measured by DNMS with trialunique objects. Monkeys with this lesion were severely impaired

Table 3. Group mean performance scores on DNMS

\begin{tabular}{lllllllll} 
& \multicolumn{9}{l}{ Delays (sec) } & \multicolumn{2}{l}{ List lengths } & Average \\
\cline { 2 - 5 } Group & 30 & 60 & 120 & 3 & 5 & 10 & 67.3 \\
\hline Rh & 76 & 71 & 67 & 69 & 61 & 60 & 59.5 \\
A + H & 68 & 57 & 60 & 58 & 59 & 55 & 60.5 \\
A + Rh & 68 & 60 & 58 & 62 & 59 & 56 & 84.3 \\
H + Rh & 89 & 86 & 85 & 88 & 83 & 75 &
\end{tabular}

Data are from Mishkin (1978; $\mathrm{A}+\mathrm{H}$ ), Murray and Mishkin (1986; $\mathrm{A}+\mathrm{Rh}, \mathrm{H}+\mathrm{Rh})$, and the present study (Rh) Conventions are as in Table 2 . Note that the $A+R h$ and $H+R h$ groups were composed of cynomolgus monkeys, whereas the $R h$ and $A+H$ groups were composed of rhesus monkeys. On the performance test (three longer delays and three list lengths), group $R h$ scored significantly better than both groups $\mathrm{A}+\mathrm{H}$ and $\mathrm{A}+\mathrm{Rh}$, and group II $+\mathrm{Rh}$ performed significantly better than each of the other three groups (Newman-Keuls test, $p<0.05$ for all comparisons). The relatively good performance of group $\mathrm{H}+\mathrm{Rh}$ is now seen to be due to the sparing, in this group only, of the rostralmost portion of rhinal cortex. 


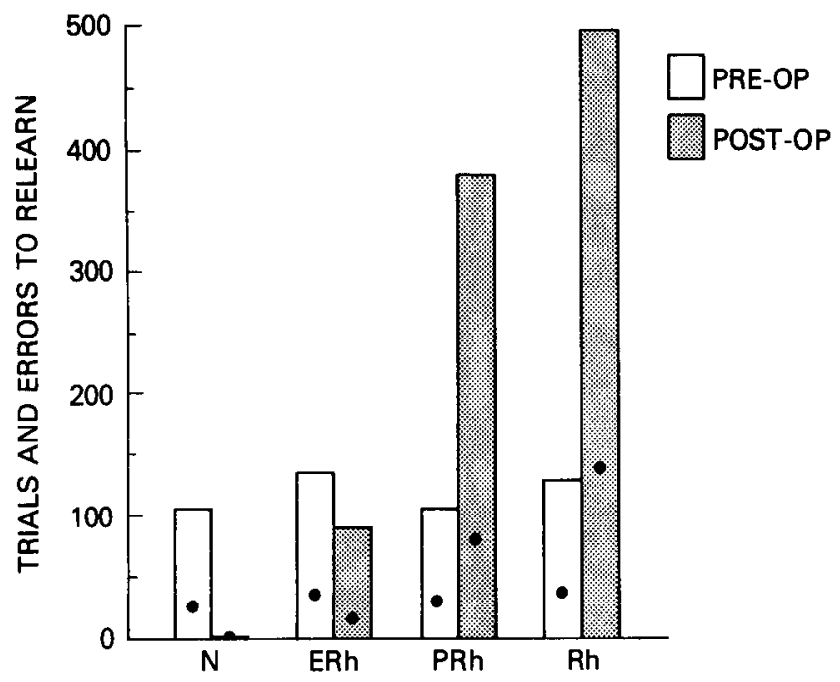

Figure 8. Pre- and postoperative learning scores on DNMS. The height of the bars indicates the mean number of trials for each group to learn or relearn, and the solid circles indicate the mean number of errors. $N$, unoperated control group $(N=4) ; E R h$, group with entorhinal cortex lesions $(N=4) ; P R h$, group with perirhinal cortex lesions $(N=4) ; R h$, group with rhinal cortex lesions $(N=7)$.

both in postoperative relearning of the basic nonmatching principle and in the subsequent performance test with extended delays and lists. These results lead to a different conclusion from the one drawn earlier by Murray and Mishkin (1986) from their comparison of the effects of rhinal cortex lesions combined with either amygdalectomy $(\mathrm{A}+\mathrm{Rh})$ or hippocampectomy $(\mathrm{H}+\mathrm{Rh})$. That is, it now appears likely that the $\mathrm{A}+\mathrm{Rh}$ lesion was far more disruptive than the $\mathrm{H}+\mathrm{Rh}$ lesion not because the former lesion, unlike the latter, was functionally equivalent to a combined amygdalohippocampectomy, but rather, as proposed in the introductory remarks, because the $\mathrm{A}+\mathrm{Rh}$ lesion included more of the rhinal cortex. If so, an $\mathrm{H}+\mathrm{Rh}$ lesion that also included more of the rhinal cortex should likewise produce a severe recognition memory loss. This prediction has recently been confirmed (Meunier et al., 1993; Zola-Morgan et al., 1993).

On relearning the DNMS rule, ablations of the perirhinal cortex alone produced nearly as severe a deficit as that induced by the combined lesions, whereas ablations of the entorhinal cortex alone had no significant effect. On the performance test, however, the effects of perirhinal ablations were not as severe as those of the combined lesions, and here the entorhinal ablations induced a mild, but significant, impairment. Apparently, although the perirhinal portion of the combined rhinal cortex lesion was largely if not wholly responsible for the relearning deficits after the combined ablation, the entorhinal damage did contribute significantly to the performance deficit. These conclusions are considered in greater detail below.

\section{Effects of rhinal cortex lesions}

Rhinal cortex lesions yielded severe impairment in visual recognition memory as evidenced by both the retardation in relearning and the low scores on the performance test. That the impairment is indeed in memory and not simply a difficulty in relearning or performing the DNMS rule is indicated by the significant interaction between the effects of lesion and delay. [Ringo (1991) recently challenged this use of the lesion-by-delay interaction term, in part on the argument that data from the

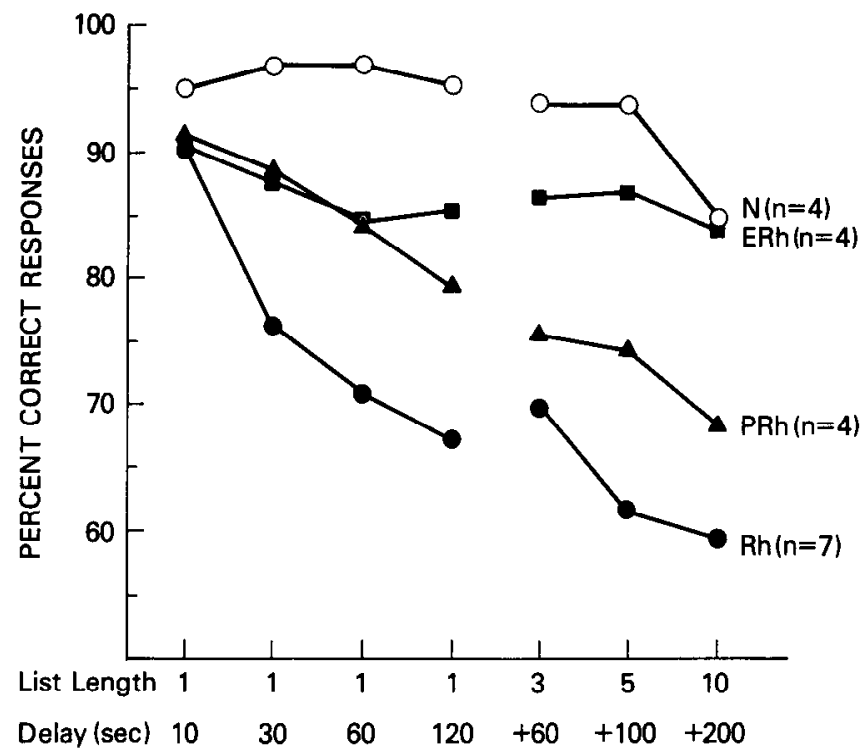

Figure 9. Postoperative performance on DNMS. Curves show the mean score for each group when increasingly longer delays were imposed between sample and choice test (left) or when lists of items were presented (right). Note that the increasing number of list items was confounded with increasing delays between sample and choice. The approximate delay between each sample and choice for a given list length is indicated on the abscissa. Abbreviations are as in Figure 8.

initial $(10 \mathrm{sec})$ delay is not a pure performance measure because it is based on the criterion run during the relearning of the rule and should therefore be excluded from the ANOVA. However, medial temporal ablation studies in which short and long delays were intermixed pseudorandomly within sessions after the animals had (re)learned the DNMS rule (Overman et al., 1990; Alvarez-Royo et al., 1992) have still yielded a significant lesion by delay interaction, strongly supporting the interpretation of a significant memory loss.]

Although the rhinal lesions in the various cases encroached slightly on area TG, posterior TE, and the parahippocampal gyrus, this extrarhinal damage is unlikely to account for the deficits because, first, these slight encroachments did not occur consistently, and second, even complete ablations of one or another of these areas fail to disrupt performance on visual recognition tests (Murray and Mishkin, 1984; Yaginuma, 1990).

The marked impairment in relearning after rhinal cortex lesions is consistent with a recent finding (Gaffan and Murray, 1992) that cynomolgus monkeys with similar lesions failed to learn delayed matching-to-sample (DMS) with double sample presentations within the training limit of 510 trials. (In that study, the DMS training was given only postoperatively and after administration of another task, which may account in part for the apparently greater relearning deficit observed there.)

With regard to the performance test, the impairment after rhinal cortex lesions was far more severe than that found previously after separatc removals of the hippocampus or the amygdala, even though each of these ablations included about one-half of the rhinal cortex (damage to the caudal half accompanying hippocampectomy and to the rostral half accompanying amygdalectomy; see below). Of these three components of the medial temporal lobe, it is clear now that the rhinal cortex is by far the most important one for recognition memory. At the same time, the impairment after rhinal cortex lesions did not 
quite reach the magnitude of that seen previously after either combined ablations of the amygdaloid complex and hippocampal formation or combined ablations of the amygdala plus rhinal cortex (Table 3). The explanation for this might be that the latter two groups sustained more complete damage to the rhinal cortex than did the $\mathrm{Rh}$ group in the present study. This is almost certainly the case for the entorhinal field, which is usually completely removed after combined limbic lesions, whereas in the present study the most medial part of the entorhinal cortex was consistently spared. It may also be the case that the inclusion of piriform and periamygdaloid cortex contributed to the greater impairment in the two groups with combined limbic lesions. Whatever the explanation for these group differences, it is now clear that the relatively mild impairment found earlier in the $\mathrm{H}+\mathrm{Rh}$ group (see Table 3 ) is attributable to the sparing of the rostralmost portion of rhinal cortex in that group

The conclusion that extensive ablation of rhinal cortex alone is sufficient to produce a marked deficit in recognition memory is supported by recent behavioral work in rodents, in which rhinal cortex lesions have been found to yield a severe impairment in olfactory DNMS (Otto and Eichenbaum, 1992) and in cue memory in the eight-arm radial maze, each arm having been covered with a different material, thereby providing distinctive tactual and visual cues to guide choices (Jarrard, 1993).

\section{Effects of perirhinal cortex lesions}

As indicated above, both the relearning and performance deficits observed after the rhinal cortex lesion appear to have been due largely to the perirhinal component of that lesion. These findings are consistent with those of other investigators who have examined the effects on visual recognition of cooling or ablating the inferior temporal gyrus, which includes the perirhinal cortical field (Horel et al., 1984, 1987; Yaginuma, 1990), or of ablating the perirhinal plus parahippocampal cortex (Zola-Morgan et al., 1989b, 1993; Beason et al., 1990; Suzuki et al., 1993). Since even the complete parahippocampal lesions that accompany hippocampectomy do not markedly disrupt visual recognition, it seems unlikely that the incomplete parahippocampal damage in the study by Zola-Morgan et al. (1989b) contributed substantially to the severe deficit they observed. On the other hand, the perirhinal component of the lesion in that study extended more laterally than the perirhinal lesions in the present experiment, and, furthermore, the white matter subjacent to the perirhinal cortex was intentionally severed in the animals studied by Zola-Morgan et al. in order to eliminate any remaining cortical input to the entorhinal cortex. Both of these differences may well have contributed to the apparently greater deficit found in that study than in the present PRh group. In short, numerous studies have now provided converging evidence regarding the importance of the perirhinal field in visual recognition memory in monkeys.

\section{Effects of entorhinal cortex lesions}

Because the ERh group exhibited no impairment in relearning DNMS, and because the PRh group exhibited a relearning impairment roughly equivalent to that of the $\mathrm{Rh}$ group, the contribution of the entorhinal removal to the latter's deficit would appear to be negligible. However, the two animals in the $\mathrm{Rh}$ and PRh groups that received two-stage operations (Rh-7 and $\mathrm{PRh}-4)$ exhibited the best retention within their respective groups, so we cannot rule out the possibility that monkeys in group $\mathrm{ERh}$, if given one-stage instead of two-stage operations, would then have shown a significant relearning impairment. (Both Rh-7 and PRh-4 were at the mean of their respective groups on the performance test, so the same argument probably does not apply to this measure.) On the performance test, the ERh group did show a significant, albeit mild, deficit. Histological findings indicate that, whereas the lesions in the PRh group were successfully limited to the perirhinal cortex, those in the ERh group consistently included partial damage to the perirhinal field ( $43 \%$ of this field on average). The mild impairment observed in group ERh could thus have been due at least in part to this perirhinal damage. Indeed, the two ERh animals (cases 2 and 4) with the greater perirhinal damage (approximately $60 \%$ as compared to approximately $30 \%$ for the two others) obtained performance scores that were about $10 \%$ lower than those of the two others. Nevertheless, an effect from the entorhinal damage cannot be entirely excluded. Because the rhinal cortex lesions produced a greater deficit on the performance test than perirhinal lesions alone, and because this greater deficit cannot be attributed to more complete perirhinal damage in the Rh group (see Table 1), the additional entorhinal damage could well be responsible. This interpretation is consistent with the findings of Beason et al. (1990) and of Leonard et al. (1992), who found that monkeys with entorhinal lesions were impaired relative to controls on a delayed recognition-span task and mildly impaired relative to controls on DNMS, respectively.

Because entorhinal cortex is the major relay for cortical sensory inputs to the hippocampus, the relatively mild deficit after entorhinal lesions alone may seem surprising. It must be noted again, however, that the ERh lesions were incomplete, sparing on average $25 \%$ of the total extent of the entorhinal cortex. More complete lesions may have produced greater impairment, and, indeed, one explanation offered earlier for the greater impairment after $\mathrm{A}+\mathrm{H}$ and $\mathrm{A}+\mathrm{Rh}$ lesions than after $\mathrm{Rh}$ lesions (see Table 3) was the more complete removal of entorhinal cortex in the former groups. Even complete hippocampal lesions, however, also lead to only a mild recognition deficit (Mishkin, 1978; Murray and Mishkin, 1984). Thus, any larger contribution than this from entorhinal cortex would necessarily implicate entorhinal projections to structures other than the hippocampus.

\section{Neural substrates of visual recognition memory}

Earlier studies (Zola-Morgan et al., 1982; Murray and Mishkin, 1984; Saunders et al., 1984) had appeared to support the suggestion (Mishkin, 1978) that combined ablations of the amygdaloid complex and hippocampal formation, removals that also included portions of the subjacent rhinal cortex, produced a severe impairment in visual recognition. The demonstration that ablation of the rhinal cortex alone is sufficient to produce a deficit almost as severe is consistent with a reinterpretation of the earlier findings, namely, that within the medial temporal region, the rhinal cortex may be the only critical substrate for recognition memory, at least over the intervals examined in these studies. One problem with this reinterpretation is that although the entorhinal cortex was deliberately included in the combined amygdalohippocampal lesions, the perirhinal cortex was not. Nevertheless, it is likely that critical efferents of the perirhinal cortex coursing adjacent to the amygdala were transected by the aspiration lesions (Murray, 1992), thus rendering the perirhinal cortex dysfunctional. This idea is consistent with recent data indicating that neither electrolytic nor excitotoxic lesions of the amygdala, made alone or in combination with 
other structures (Zola-Morgan et al., 1989a; E. Gaffan and Murray, 1991, unpublished observations), nor selective lesions of the hippocampus (Clower et al., 1991) disrupt recognition as much as aspiration lesions of the same structures that include some of the subjacent rhinal cortex. One alternative interpretation of all the foregoing findings has, however, not yet been ruled out. The alternative is that lesions restricted to the rhinal cortex, on the one hand, and to the amygdala and hippocampus, on the other hand, may each be sufficient to yield severe impairment. While the present study demonstrates that removals of the rhinal cortex are indeed sufficient, the effects on recognition of combined amygdala and hippocampal removals that spare the rhinal cortex remain to be tested.

The rhinal cortex receives direct projections from neocortical areas serving all the sensory modalities (Jones and Powell, 1970; Turner et al., 1980; Friedman et al., 1986; Insausti ct al., 1987; Suzuki and Amaral, 1990; Martin-Elkins and Horel, 1992). This region projects, in turn, to both the amygdala and hippocampus (Van Hoesen and Pandya, 1975a,b; Saunders and Rosene, 1988), structures that also receive direct projections from area TE and the parahippocampal gyrus (Herzog and Van Hoesen, 1976; Van Hoesen et al., 1979; Yukie et al., 1990; Webster et al., 1991). Because the recognition deficit after rhinal cortex lesions is far more severe than that which follows complete hippocampectomy, the rhinal cortical contribution cannot be mediated solely by entorhinal projections to the hippocampus, but, rather, must be mediated through other pathways. The rhinal cortex projects directly to the mediodorsal nucleus of the thalamus via the ventral amygdalofugal pathway (Aggleton and Mishkin, 1984; Aggleton et al., 1986; Russchen et al., 1986; Gower, 1989), to the anterior thalamic nuclei and mammillary bodies via the fornix (Saunders, 1983; Rosene and Saunders, 1987), and to other cortical regions known to be involved in visual recognition memory, such as the ventromedial prefrontal cortex (Suzuki and Amaral, 1990), via pathways such as the uncinate fasciculus. Thus, an earlier finding that severe recognition impairments follow combined but not separate transections of the fornix and amygdalofugal pathways (Bachevalier et al., 1985), originally interpreted as being due to disconnection of the medial diencephalon and ventromedial prefrontal cortex from the amygdala and hippocampus, may be due in addition, or even instead, to disconnection of these structures from the rhinal cortex.

\section{References}

Aggleton JP, Mishkin M (1984) Projections of the amygdala to the thalamus in the cynomolgus monkey. J Comp Neurol 222:56-68.

Aggleton JP, Desimone R, Mishkin M (1986) The origin, course, and termination of the hippocampo-thalamic projections in the macaque. J Comp Neurol 243:409-421.

Alvarez-Royo P, Zola-Morgan S, Squire LR (1992) Impairment of long-term memory and sparing of short-term memory in monkeys with medial temporal lobe lesions: a response to Ringo. Behav Brain Res 52:1-5.

Amaral DG, Insausti R, Cowan WM (1987) The entorhinal cortex of the monkey. I. Cytoarchitectonic organization. J Comp Neurol 264: 326-355.

Bachevalier J, Parkinson JK, Mishkin M (1985) Visual recognition in monkeys: effects of separate vs. combined transection of fornix and amygdalofugal pathways. Exp Brain Res 57:554-561.

Beason LL, Moss MB, Rosene DL (1990) Effects of entorhinal, parahippocampal, or basal forebrain lesions on recognition memory in the monkey. Soc Neurosci Abstr 16:617.

Boussaoud D, Desimone R, Ungerleider LG (1991) Visual topography of area TEO in the macaque. J Comp Neurol 306:554-575.

Clower RP, Alvarez-Royo P, Zola-Morgan S, Squire LR (1991) Rec- ognition memory impairment in monkeys with selective hippocampal lesions. Soc Neurosci Abstr 17:140.

Fiorani M Jr, Gattass R, Rosa MGP, Sousa APB (1989) Visual area MT in the Cebus monkey: location, visuotopic organization, and variability. J Comp Neurol 287:98-1 18.

Friedman DP, Murray EA, O'Neill JB, Mishkin M (1986) Cortical connections of the somatosensory fields of the lateral sulcus of macaques: evidence for a corticolimbic pathway for touch. J Comp Neurol 252:323-347.

Gaffan D (1974) Recognition impaired and association intact in the memory of monkcys after transection of the fornix. J Comp Physiol Psychol 86:1100-1109.

Gaffan D, Murray EA (1992) Monkeys with rhinal cortex lesions succeed in object discrimination learning despite 24-hour intertrial intervals and fail at matching to sample despite double sample presentations. Behav Neurosci 106:30-38.

Gaffan EA, Murray EA (1991) Effects of anterior rhinal cortical lesions on delayed nonmatching-to-sample in rhesus monkeys. Soc Neurosci Abstr 17:338.

Gattass R, Gross CG (1981) Visual topography of striate projection zone (MT) in posterior superior temporal sulcus of the macaque. $J$ Neurophysiol 46:621-638.

Gower EC (1989) Efferent projections from limbic cortex of the temporal pole to the magnocellular medial dorsal nucleus in the rhesus monkey. J Comp Neurol 280:343-358.

Herzog AG, Van Hoesen GW (1976) Temporal neocortical afferent connections to the amygdala in the rhesus monkey. Brain Res 115 : $57-69$.

Hodos W, Bobko P (1984) A weighted index of bilateral brain lesions. J Neurosci Methods 12:43-47.

Horel JA, Voytko ML, Salsbury KG (1984) Visual learning suppressed by cooling the temporal pole. Behav Neurosci 98:310-324.

Horel JA, Pytko-Joiner DE, Voytko ML, Salsbury K (1987) The performance of visual tasks while segments of the inferotemporal cortex are suppressed by cold. Behav Brain Res 23:29-42.

Insausti R, Amaral DG, Cowan WM (1987) The entorhinal cortex of the monkey. II. Cortical afferents. J Comp Neurol 264:356-395.

Jarrard LE (1993) On the role of the hippocampus in learning and memory in the rat. Behav Neural Biol, in press.

Jones EG, Powell TPS (1970) An anatomical study of converging sensory pathways within the cerebral cortex of the monkey. Brain 93: 793-820.

Leonard BW, Amaral D, Zola-Morgan S, Squire LR (1992) Selective entorhinal cortical lesions impair learning and memory in the monkey. Soc Neurosci Abstr 18:388.

Martin-Elkins CL, Horel JA (1992) Cortical afferents to behaviorally defined regions of the inferior temporal and parahippocampal gyri as demonstrated by WGA-HRP. J Comp Neurol 321:177-192.

Meunier M, Murray EA, Bachevalier J, Mishkin M (1990) Effects of perirhinal cortical lesions on visual recognition memory in rhesus monkeys. Soc Neurosci Abstr 16:616.

Meunier M, Hadfield W, Bachevalier J, Murray EA (1993) Effects of rhinal cortical lesions combined with hippocampectomy on visual recognition memory in rhesus monkeys. Soc Neurosci Abstr, in press.

Mishkin M (1978) Memory in monkeys severely impaired by combined but not by separate removal of amygdala and hippocampus. Nature 273:297-298.

Mishkin M, Delacour J (1975) An analysis of short-term visual memory in the monkey. J Exp Psychol 1:326-333.

Murray EA (1992) Medial temporal lobe structures contributing to recognition memory: the amygdaloid complex versus the rhinal cortex. In: The amygdala: neurobiological aspects of emotion, memory, and mental dysfunction (Aggleton JP, ed), pp 453-470. New York: Wiley-Liss.

Murray EA, Mishkin M (1984) Severe tactual as well as visual memory deficits follow combined removal of the amygdala and hippocampus in monkeys. J Neurosci 4:2565-2580.

Murray EA, Mishkin M (1986) Visual recognition in monkeys following rhinal cortical ablations combined with either amygdalectomy or hippocampectomy. J Neurosci 6:1991-2003.

Murray EA, Bachevalier J, Mishkin M (1985) Rhinal cortex: a third temporal-lobe component of the limbic memory system. Soc Neurosci Abstr 11:461.

Murray EA, Bachevalier J, Mishkin M (1989) Effects of rhinal cortical 
lesions on visual recognition memory in rhesus monkeys. Soc Neurosci Abstr 15:342.

Otto T, Eichenbaum H (1992) Complementary roles of the orbital prefrontal cortex and the perirhinal-entorhinal cortices in an odorguided delayed-nonmatching-to-sample task. Behav Neurosci 106: $762-775$.

Overman WH, Ormsby G, Mishkin M (1990) Picture recognition vs. picture discrimination learning in monkeys with medial temporal removals. Exp Brain Res 79:18-24.

Ringo JL (1991) Memory decays at the same rate in macaques with and without brain lesions when expressed in d' or arcsine terms. Behav Brain Res 42:123-134.

Rosene DL, Saunders RC (1987) The subcortical projections of the entorhinal cortex in the rhesus monkcy. Soc Ncurosci Abstr 13:886.

Russchen FT, Amaral DG, Price JL (1986) The afferent input to the magnocellular division of the mediodorsal thalamic nucleus in the monkey Macaca fascicularis. J Comp Neurol 256:175-210.

Saunders RC (1983) Experiments on memory involving the fornixmammillary system. D.Phil. thesis, Oxford University.

Saunders RC, Rosene DL (1988) A comparison of the efferents of the amygdala and the hippocampal formation in the rhesus monkey. I. Convergence in the entorhinal, prorhinal, and perirhinal cortices. J Comp Neurol 271:153-184.

Saunders RC, Murray EA, Mishkin M (1984) Further evidence that amygdala and hippocampus contribute equally to recognition memory. Neuropsychologia 22:785-796.

Suzuki WA, Amaral DG (1990) The organization of cortical inputs to the perirhinal (areas 35 and 36) and parahippocampal (areas TF and $\mathrm{TH}$ ) cortices in the monkey. Soc Neurosci Abstr 16:53.

Suzuki WA, Zola-Morgan S, Squire LR, Amaral DG (1993) Lesions of the perirhinal and parahippocampal cortices in the monkey produce long lasting memory impairments in the visual and tactual modalities. $\mathrm{J}$ Neurosci, in press.

Turner BH, Mishkin M, Knapp M (1980) Organization of the amygdalopetal projections from modality-specific cortical association areas in the monkey. J Comp Neurol 191:515-543.
Van Hoesen GW, Pandya DN (1975a) Some connections of the entorhinal (area 28) and perirhinal (area 35) cortices of the rhesus monkey. I. Temporal lobe afferents. Brain Res 95:1-24.

Van Hoesen GW, Pandya DN (1975b) Some connections of the entorhinal (area 28) and perirhinal (area 35) cortices of the rhesus monkey. III. Efferent connections. Brain Res 95:39-59.

Van Hoesen GW, Rosene DL, Mesulam M-M (1979) Subicular input from temporal cortex in the rhesus monkey. Science 205:608-610.

von Bonin G, Bailey P (1947) The neocortex of Macaca mulatta. Urbana, IL: University of Illinois.

Webster MJ, Ungerleider LG, Bachevalier J (1991) Connections of inferior temporal areas TE and TEO with medial temporal lobe structures in infant and adult monkeys. J Neurosci 11:1095-1116.

Yaginuma S (1990) Functional subdivisions of area TE of the inferotemporal cortex in the monkey. In: Vision, memory, and the temporal lobe (Iwai E, Mishkin M, eds), pp 29-41. New York: Elsevier.

Yukie M, Takeuchi H, Hasegawa Y, Iwai E (1990) Differential connectivity of inferotemporal area TE with the amygdala and the hippocampus in the monkey. In: Vision, memory, and the temporal lobe (Iwai E, Mishkin M, eds), pp 129-165. New York: Elsevier.

Zola-Morgan S, Squire LR, Mishkin M (1982) The neuroanatomy of amnesia: amygdala-hippocampus versus temporal stem. Science 218 : $1337-1339$.

Zola-Morgan S, Squire LR, Amaral DG (1989a) Lesions of the amygdala that spare the adjacent cortical regions do not impair memory or exacerbate the impairment following lesions of the hippocampal formation. J Neurosci 9:1922-1936.

Zola-Morgan S, Squire LR, Amaral DG, Suzuki WA (1989b) Lesions of the perirhinal and parahippocampal cortex that spare the amygdala and hippocampal formation produce severe memory impairment. J Neurosci 9:4355-4370.

Zola-Morgan S, Squire LR, Clower RP, Rempel NL (1993) Damage to the perirhinal cortex exacerbates memory impairment following lesions to the hippocampal formation. J Neurosci 13:251-265. 